\title{
Impact of liquid droplets on granular media
}

\author{
G. Delon, ${ }^{*}$ D. Terwagne, S. Dorbolo, N. Vandewalle, and H. Caps \\ GRASP, Physics Department B5, Université de Liège, B4000-Liège, Belgium
}

(Received 15 March 2011; published 25 October 2011)

\begin{abstract}
The crater formation due to the impact of a water droplet onto a granular bed has been experimentally investigated. Three parameters were tuned: the impact velocity, the size of the droplet, and the size of the grains. The aim is to determine the influence of the kinetic energy on the droplet pattern. The shape of the crater depends on the kinetic energy at the moment the droplet starts to impact the bed. The spreading and recession of the liquid during the impact were carefully analyzed from the dynamical point of view, using image analysis of high-speed video recordings. The different observed regimes are characterized by the balance between the impregnation time of the water by the granular bed by the water and the capillary time responsible for the recession of the drop.
\end{abstract}

DOI: 10.1103/PhysRevE.84.046320

PACS number(s): 47.55.D-, 83.80.Fg, 47.55.nd

\section{INTRODUCTION}

The impact of liquid droplets or solid objects on a liquid pool is probably the most prolific topic in the physics of fluids. It is rather interesting to reread the article by Worthington published in the late 19th century [1]. The subject is the splash of droplets. The paper is one of the first works that concerns fast imaging and the analysis of the impact of a droplet on a solid. Moreover, Worthington considers, in the same article, the impact of a solid sphere on a pool of liquid. The crux is that all these subjects are still considered in recent papers. The impact of a droplet on a pool of liquid has generated a large number of publications about corona formation [2], the bell formed by the impact of large droplets [3], the trapping of small bubbles [4], and so on. The splash of a droplet on a solid surface is also a very active subject of research, finding application in numerous industrial areas: painting, self-cleaning windows, impact on airplane wings, and agricultural pulverization [5].

From the point of view of fundamental science, the splash of a droplet on a solid surface has been reconsidered thanks to the fine control of the surface texture and the chemical nature now available. Custom-made Fakir surfaces have been intensively investigated since the splash can be really controlled [6,7]. Finally, it is remarkable that the impact of a solid sphere on a pool is also at the cutting edge of research. For example, Worthington considered solid spheres that were preliminarily sandpapered in order to texture them. The problem has been reconsidered by Aristoff and Bush [8].

Granular materials are known to exhibit behaviors that can be assimilated to solid, liquid, or gas according to the constraints. In 2004, Lohse et al. considered the impact of a solid sphere on a fluidized granular lid [9]; after the impact, a vertical jet of grains, the so-called Worthington jet, is observed. In 2009, Deboeuf et al. studied grain ejection following the impact of a solid bead in sand [10]. In the present paper, a case from everyday life is approached: the impact of rain droplets on a beach. The behavior on hitting a granular layer is more complex than for a solid or fluid surface. The sand may be considered as soft matter and, consequently, the sand behavior depends on the energy of the droplet. The problem is defined by a large number of parameters, namely, the droplet size, the

\footnotetext{
*giles.delon@ulg.ac.be
}

fluid viscosity, the fluid surface tension, the impact velocity, and the grain size, shape, and volume fraction.

The impact of drops on sand has recently been studied independently by Dalziel and Steaton [11] and by Katsuragi [12]. Both studies concern the final shape of the crater. While a repertoire of the possible morphologies for the craters is proposed in [11], a scaling law for the radius of those craters is proposed in [12]. The shape of the crater and that of the surrounding rim have also been measured by use of the light transmission method [12]. The last study, however, is limited in the range of heights of the fall, mainly because of the hydrophobic nature of the sand used in this study, which favors the atomization of the droplet, as studied in [13]. Above a given height of fall, the droplet bursts into smaller parts.

In this paper, we focus on the influence of the radius of the droplet, the granulometry of the sand, and the height of fall. We tuned the energy of the droplet without changing the chemistry (e.g., wetting properties, fluid viscosity) in order to focus on the mechanical dynamics. Starting from the static final shape of the craters, we go through the dynamics of the spreading and recession of the droplet by mean of high-speed recordings. Different regimes are observed and explained. Scaling laws are then proposed.

\section{EXPERIMENTAL SETUP}

The experiment consists in dropping a water droplet onto a granular layer. We consider only pure water with a surface tension $\sigma=72 \mathrm{mN} / \mathrm{m}$, a viscosity $\eta=1.002 \mathrm{mPa} \mathrm{s}$, and a density $\rho=1000 \mathrm{~kg} / \mathrm{m}^{3}$ at a temperature $T=20^{\circ} \mathrm{C}$. The droplets are produced using a Hamilton syringe. The pendant droplet detaches from the needle when the capillary forces cannot balance the height of the droplet any longer, allowing a good reproducibility of the droplet size: the radius of the droplet is proportional to the cube root of the needle radius [14]. The diameters $D$ of the used needles are $0.4,0.8$, and $1 \mathrm{~mm}$; the droplet radii obtained are then 1.152 (denoted $\alpha$ ), $1.35(\beta)$, and $1.86 \mathrm{~mm}(\gamma)$, respectively.

The height of the drop, $h$, was progressively increased in steps of $15 \mathrm{~mm}$. A high-resolution picture of the shape of the generated craters was taken using a digital camera. A preliminary calibration allowed measurement of the size of the craters (see the next section). In a second set of experiments, a high-speed video camera (IDT N3) was used to observe 
TABLE I. Summary of droplet radii $r$, glass bead diameters $d$, and porosity $\kappa$ of each kind of sand.

\begin{tabular}{lcccc}
\hline \hline Droplet & $\begin{array}{c}r \\
(\mathrm{~mm})\end{array}$ & Sand & $\begin{array}{c}\langle d\rangle \\
(\mu \mathrm{m})\end{array}$ & $\begin{array}{c}\kappa \\
\left(\mu \mathrm{m}^{2}\right)\end{array}$ \\
\hline$\alpha$ & 1.35 & $A$ & 55 & 444 \\
$\beta$ & 1.53 & $B$ & 120 & 580 \\
$\gamma$ & 1.86 & $C$ & 300 & 3628 \\
\hline \hline
\end{tabular}

the impacts at 5000 frames per second (fps). This allowed an accurate analysis of the spreading and recession dynamics of the water droplet impacting the granular bed. For that purpose, the high-speed camera was oriented at $60^{\circ}$ from the granular bed. The water was colored in order to enhance the contrast.

The sand was made of micrometric glass beads (Silibeads). Three grain sizes were used: sand $A(\langle d\rangle=105 \pm 65 \mu \mathrm{m})$, sand $B(\langle d\rangle=120 \pm 30 \mu \mathrm{m})$, and sand $C(\langle d\rangle=300 \pm$ $100 \mu \mathrm{m})$. The depth of the sand layer was fixed to $20 \mathrm{~mm}$. We observed that if the depth is less than $10 \mathrm{~mm}$, the bottom influences the impact dynamics. The porosity $\kappa$ is defined as the characteristic section of a pore in between grains of sand. We estimate $\kappa$ by considering three spherical grains of radius $R$ forming a compact triangle; the section between is equal to $(\sqrt{3}-\pi / 2) R^{2}$. Table I summarizes the radius of the droplets and the size and porosity of each kind of sand we used.

\section{EXPERIMENTAL RESULTS}

In order to evaluate the impact energy, the impacting velocity of the droplets has been measured as a function of the height of fall $h$. The results corresponding to a drop of kind $\beta$ are shown in the inset of Fig. 2 below. The velocity is found to follow a free fall law until the height of the drop $h=800 \mathrm{~mm}$. The maximum drop velocity that was reached is close to $4 \mathrm{~m} / \mathrm{s}$. Accounting for the mass of the droplet and its velocity when considering the impact energy, the Weber number comparing inertia and surface tension is defined as

$$
\mathrm{We}=\frac{\rho v^{2} D}{\sigma},
$$

where $D=2 r$ is the drop diameter, $\sigma$ is the surface tension, and $\rho$ is the mass per volume unit of the water.

In the following, this Weber number is used as a control parameter for studying the crater morphology (Sec. III A) and the impact dynamics (Sec. III B).

\section{A. Crater morphology}

Figure 1 presents seven samples of the craters resulting from the impacts of a colored droplet $\beta$ on a layer of sand $B$. From left to right, the height of fall is increased in steps of $12 \mathrm{~mm}$. These snapshots are representative of the possible crater morphologies. In order to describe the shapes of these craters, we define two characteristic diameters: (i) a large crater with a diameter $D_{\max }$, which is the maximum area disturbed by the droplet, and (ii) the residual droplet that soaked the grains at the center, with a diameter $D_{\text {final }}$. In the first snapshot of Fig. 1, both characteristic diameters can be easily identified. For the smallest and largest We values, $D_{\max }$ is of the order of magnitude of $D_{\text {final }}$, while for intermediate We values the residual droplet is observed to be significantly smaller than the global crater.

The latter observation is confirmed by Fig. 2, where the ratio between the extension of the crater $D_{\max }$ and the droplet size $D$ is plotted as a function of the Weber number for the droplet $\beta$ on sand. The monotonic growth is well fitted by the power law

$$
\frac{D_{\max }}{D} \sim \mathrm{We}^{1 / 4} .
$$

This law is reminiscent of the impact of a droplet onto a solid [6], as already stated in [12]. This is a clear signature that the granular material may be pictured as a solid during the first part of the impact. It should, however, be noted that the prefactor to this law differs from that in [6] and is lower in our case. This result might be related to the energy loss in granular transport during crater formation.

The ratio $D_{\max } / D_{\text {final }}$ has been plotted as a function of We in Fig. 3 for droplets $\beta$ on sand $B$. At low We values, the ratio is close to unity and then increases linearly. A maximum occurs close to We $=200$. Then the $D_{\max } / D_{\text {final }}$ ratio decreases toward a value asymptotically close to unity at large We values. We claim that the different behaviors can be interpreted in terms of a competition between the characteristic time needed for the drop to expand during the splash and the time needed for the liquid composing the drop to be imbibed by the sand layer, as will be discussed in detail in Sec. III B.

At low We values, the recession time of the drop is smaller than the impregnation time. The droplet thus retracts into a shape that looks like a donut (see the second snapshot in Fig. 1). A depression can be observed in the center of the residual droplet, forming a torus. This depression is initiated by a vortex ring, as observed in [15]. For We values larger than $\mathrm{We} \approx 200$,

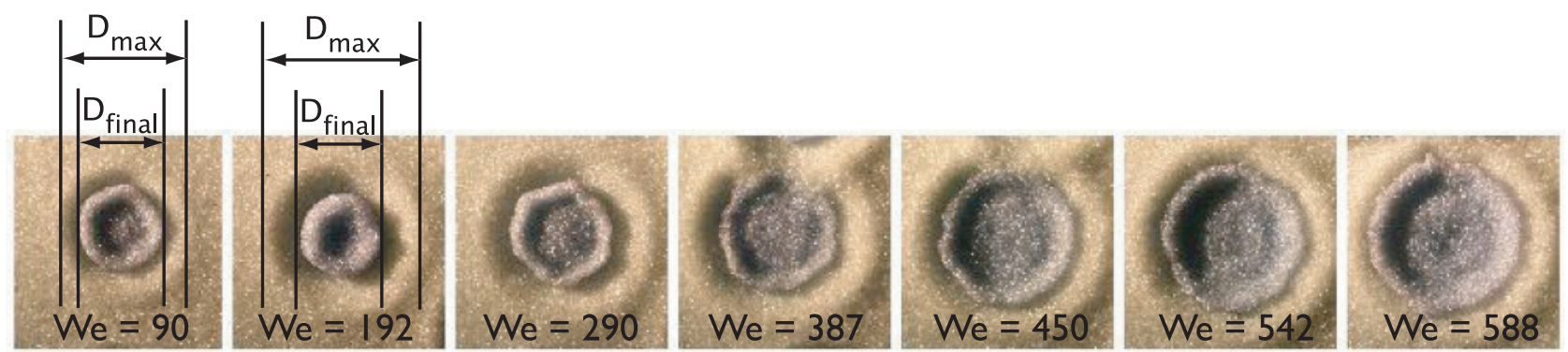

FIG. 1. (Color online) Different craters given by water droplets (size $\beta$ ) impacting a granular layer (sand $B$ ) from different initial heights $h$. From left to right, the height of dropping is increased in steps of $0.12 \mathrm{~m}$, starting at $h=0.045 \mathrm{~m}$. 


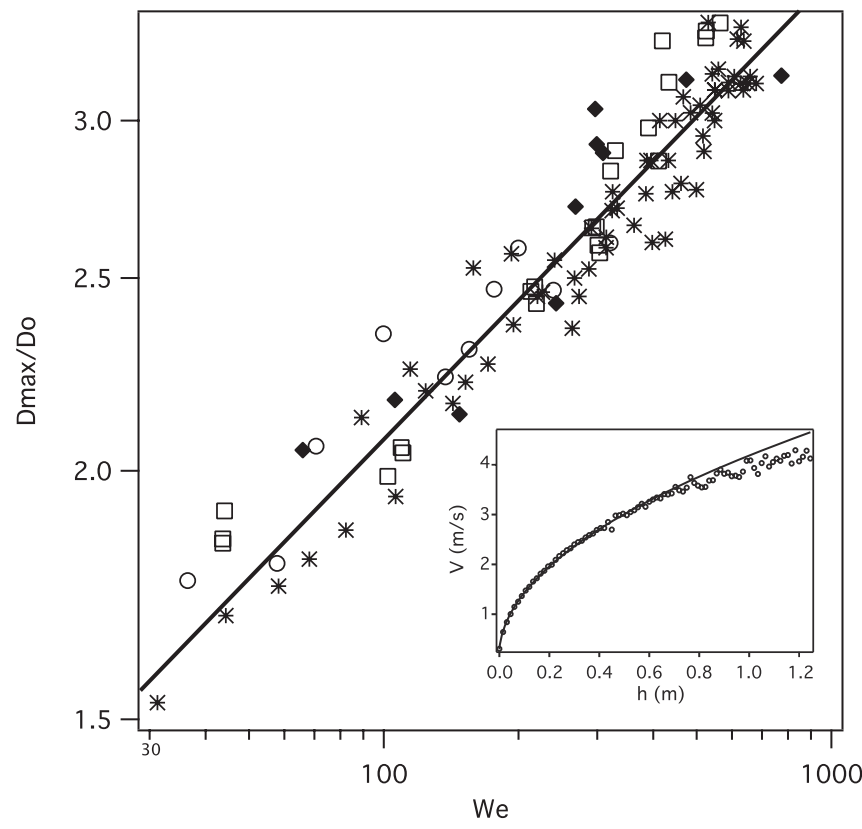

FIG. 2. Ratio between the crater extension $D_{\max }$ and the size of the impacting droplet $D$ as a function of the We number. Different drop diameters are presented: $\circ$ for drop $\alpha, *$ for drop $\beta$, and $\diamond$ for drop $\gamma$, all three impacting sand $B ; \square$ are related to drops $\beta$ on sand $A$. The solid line represents a fit using Eq. (2). The impacting velocity of a drop $\beta$ as a function of the height of fall $h$ is shown in the inset.

the impregnation is faster than the recession of the drop. The droplet does not recede. Its shape is flat and it is surrounded by a rim (see the fourth snapshot in Fig. 1). In the asymptotic regime, there is nearly no recession after the spreading because of the fast imbibition of the granular layer. The residual droplet is flat, occupies the whole crater, and has the same shape as the crater, i.e., parabolic (see the seventh snapshot in Fig. 1). This phenomenological scenario can be tested by varying the droplet size and the sand granulometry as we will show here.

In Fig. 4 , the $D_{\max } / D_{\text {final }}$ ratio is represented for the three sizes of droplet impacting sand $B$. For any drop size, the ratio tends to 1 at large We value. The large spreading of the drop is blocked by the subsequent rapid imbibition by the sand layer. For the smallest drop size, namely, $\alpha$, the maximum of the curve is much smaller than for the others. This result

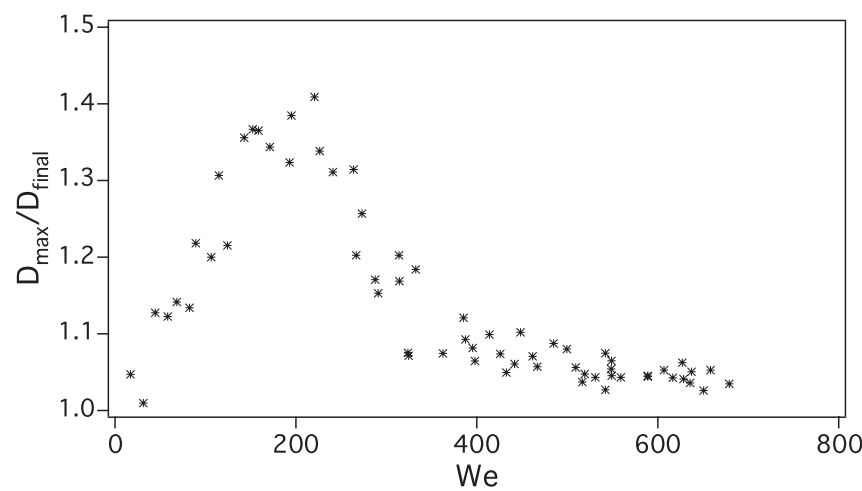

FIG. 3. Ratio between the crater diameter $D_{\max }$ and the residual droplet diameter $D_{\text {final }}$ as a function of the We number. A droplet of kind $\beta$ impacts a sand layer $B$.

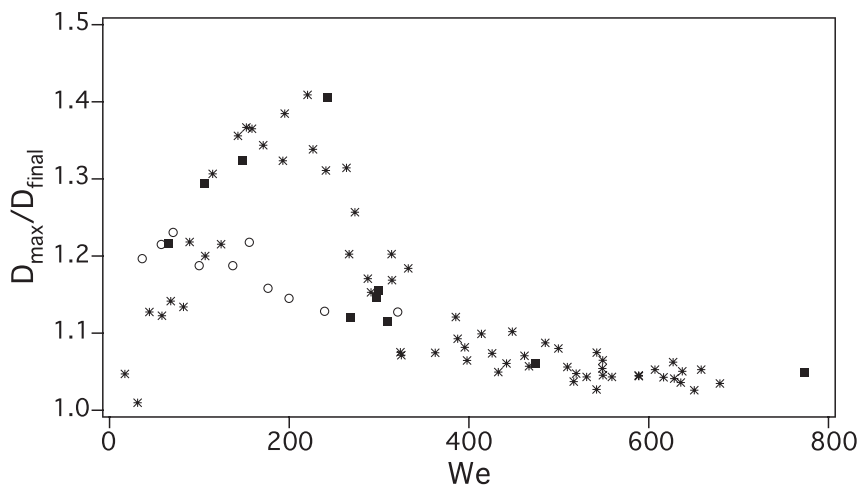

FIG. 4. Influence of the droplet size on the ratio between the extension of the crater $D_{\max }$ and the residual droplet $D_{\text {final }}$ as a function of the We number. Impacts of three droplet sizes are represented: $~$ for droplet $\alpha, *$ for $\beta$, and $\mathbf{\square}$ for $\gamma$ on sand $B$. See Table I.

emphasizes a larger contribution of capillarity in comparison to inertia. The less the droplet spreads out, the slower is the imbibition.

The ratio $D_{\max } / D_{\text {final }}$ is plotted for the three sand layer granulometries in Fig. 5. The same size of droplet is used, $\beta$. Again, whatever the considered grain size, the asymptotic regime is found for large We values. While the maximum of the curves occurs at the same We as previously for the smallest grains, it totally disappears for the largest ones $(C)$. For the largest grains, the liquid flows through the granular bed without allowing the drop to spread. Moreover, the maximum of the $D_{\text {max }} / D_{\text {final }}$ curve is much larger for the sand made with the smallest grains.

The case of the small droplet impacting the medium size sand and the case of the medium droplet impacting large-grain sand are quite similar. They evidence that the description using only the We number is not sufficient to explain the different regimes. Discrimination of the two cases would require comparison of the kinetic energy of the droplet and the energy needed for moving sand grains. On the other hand, when the grains can be transported by the impacting droplet, the same scenario occurs: a linear regime, a maximum, and an asymptotic regime are observed. The amplitude of the phenomenon is maximum when the grains are lightest (sand $A$ ).

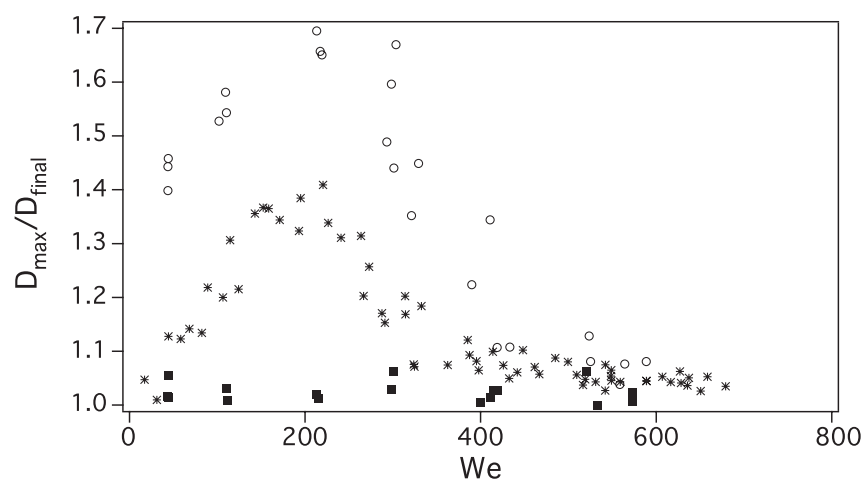

FIG. 5. Influence of the granulometry of the sand on the ratio between $D_{\max }$ and $D_{\text {final }}$ as a function of the We number. Symbols are $\circ$ for sand $A, *$ for $B$, and $\mathbf{\square}$ for $C$. 


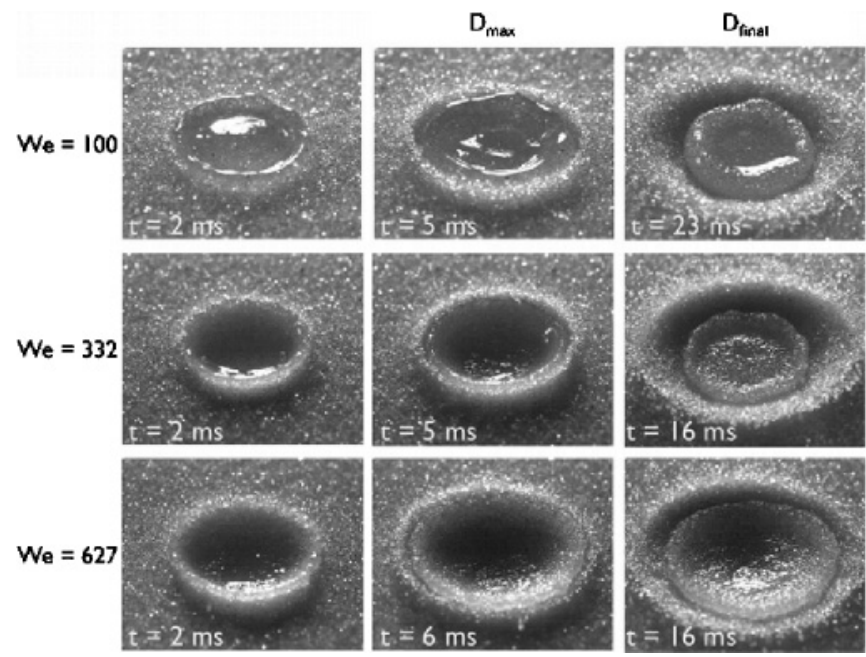

FIG. 6. Successive snapshots of the impact of a $\beta$ drop onto sand $B$. The velocity of the drop increases from the top to the bottom row. The snapshots evidence the impact, the maximal deformation, and the final shape of the residual. A video can be seen in the Supplemental Material [16].

From the crater morphology pictures, we can conclude that (i) the granular material behaves like a solid during the first part of the impact process, (ii) two regimes can be observed when the kinetic energy of the droplet is sufficient to move the grains, and (iii) the competition between the spreading and the impregnation of the drop should be relevant to describe the different regimes.

\section{B. Impact process}

The impact dynamics has been investigated by systematically analyzing high-speed movies at $5000 \mathrm{fps}$. As illustration, three snapshots corresponding to three different We values are represented in Fig. 6. The time is indicated in the different pictures ( $t=0$ is taken as the moment of impact) and corresponds to three key instants of the droplet impact. The second column concerns the spreading of the droplet on the granular medium and presents the droplet in its most squeezed state, showing the increase of $D_{\max }$ with the We number. The final shapes of the droplets after the impregnation of the water are represented in the third column. It can be seen that ejected grains are observed at large We values.

It is observed that, within the range of tested parameters, the spreading drop always accretes grains by dislodging them from the sand layer. This comes from the high speed of the spreading (typically $1 \mathrm{~m} \mathrm{~s}^{-1}$ ), which imposes an important gradient of velocity on the grains. As a result, the shear stress applied on the grain surfaces is much larger than their weight, causing them to be picked up from the sand bed. On the contrary, the ejection of grains is not observed at low We values. This ejection of grains is not caused by the liquid motion but is mainly due to the deformation of the granular bed by the impact. As the drop enters the granular medium, the compaction of the layer changes. This allows grains to be lifted out of the bed and transported in the air.

After this rapid spreading, the water retracts. Throughout this process, water tends to imbibe the granular bed. The

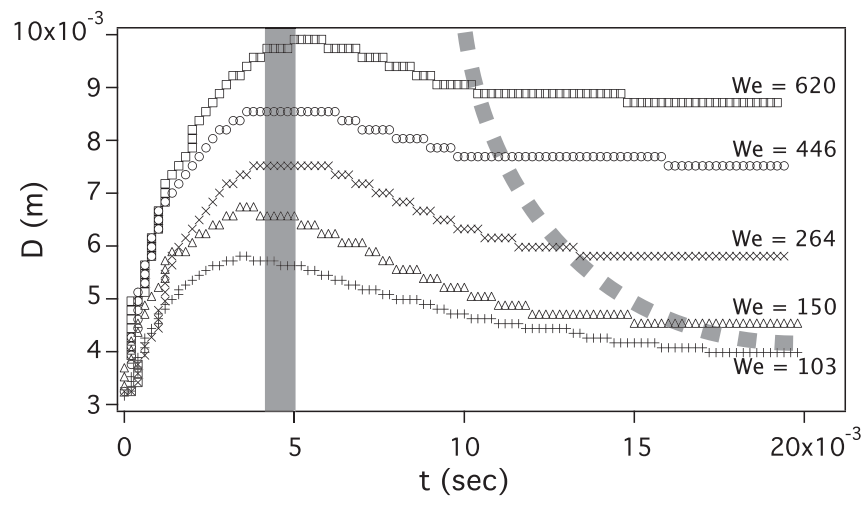

FIG. 7. Temporal evolution of the diameter of the droplet $D(t)$ (droplet $\beta$ on sand $B$ ) for We $\in[100 ; 650]$. The solid line delimits the end of the spreading while the dashed one emphasizes the end of the recession. Both are guides for the eyes.

recession process is more rapidly stopped for the higher We values because of (i) the faster imbibition of the water into the granular bed, caused by the squeezed shape of the drop, and (ii) the soaked grains, which increase the apparent viscosity of the fluid, slowing down the recession. These observations are in agreement with the measurement of the crater morphology.

In order to quantify the impact process, the diameter $D(t)$ of the droplets is reported versus time in Fig. 7 for different We values. One can observe that (i) the diameter increases monotonically (spreading time) during roughly $2 \mathrm{~ms}$; (ii) a maximum occurs for $D(t)=D_{\max }$; and (iii) $D(t)$ decreases and tends to an asymptotic value which determines $D_{\text {final }}$. Again, we can decompose the process into the spreading and the recession. The spreading is governed by the capillary time as on a solid. On the contrary, the recession seems to strongly depend on the impregnation of the granular bed.

Considering that the spreading dynamics is mainly governed by capillarity, we define the associated characteristic time as

$$
\tau_{\gamma}=\sqrt{\frac{\rho \Omega}{\sigma}},
$$

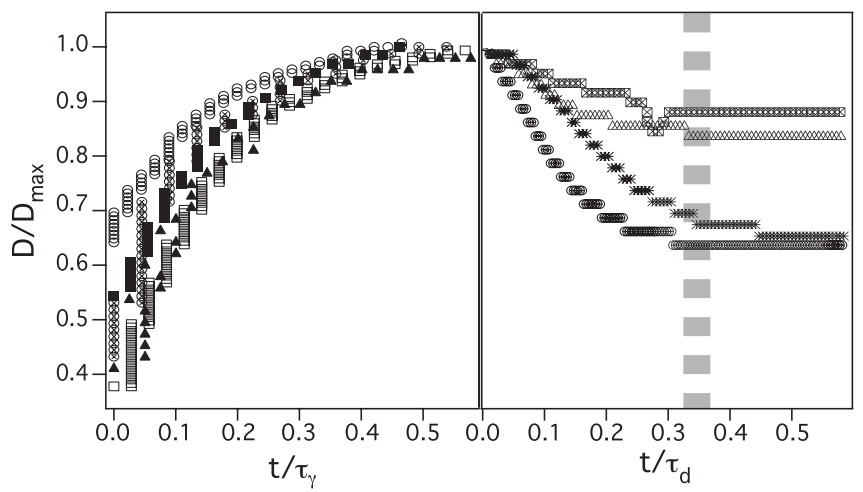

FIG. 8. Rescaling of Fig. 7 using the maximal diameter $D_{\max }$ as a characteristic length, the capillary time $\tau_{\gamma}$ for the spreading dynamics, and the impregnation time $\tau_{d}$ for the recession. Left: $*$ stands for $\alpha$ drop on sand $A$, denoted as $(\alpha, A), \square$ for $(\beta, B), \triangle$ for $(\alpha, B)$, + for $(\beta, B)$, and $\circ$ for $(\gamma, B)$. On the right, sand $B$ is impacted by $\beta$ with We $\in[100 ; 650]$. 
where $\Omega=4 \pi r^{3} / 3$ is the drop volume. This time equals 12.0 , 14.4 , and $19.3 \mathrm{~ms}$ for droplets $\alpha, \beta$, and $\gamma$, respectively. Normalization of the first part of Fig. 7 using $\tau_{\gamma}$ and the maximum diameter $D_{\max }$ is presented in Fig. 8 .

While the droplet tends to retract, the water is imbibed into the granular bed; this is the impregnation process. Due to the hydrostatic pressure below the flattened drop, water is pushed into the granular medium, seen as a porous material of permeability $\kappa$. It is then straightforward to define an imbibition time, similar to the one corresponding to Darcy's law,

$$
\tau_{d}=\frac{\Omega \eta}{\kappa \rho g D_{\max }^{2}} .
$$

Here, $\tau_{d}$ is typically equal to a few tenths of a millisecond for the droplet $\beta$ on sand $B$. The case of the sand $C$ has to be emphasized. For that grain species, $\tau_{d}$ is smaller than $\tau_{\gamma}$. Therefore, any droplet impacting that sand is imbibed immediately without spreading out, as observed in Fig. 5. The data points $D(t) / D_{\max }$ are plotted versus the nondimensional time $t / \tau_{d}$ in the right part of Fig. 8, for different We values. The collapse of the experimental data clearly reveals that the recession mechanism is governed by the impregnation.

A complete modeling of the drop dynamics is rather difficult, due to the constant interaction between capillarity and imbibition. Previous studies [17,18] proposed models for the spreading and recession of viscous drops, emphasizing the complexity of the topic, even without considering imbibition.
Moreover, in our experiments, the fluid viscosity is constantly changing with the accretion of grains inside the drop. As a consequence, the viscosity is hard to estimate. A noninvasive method for measuring or estimating this instantaneous viscosity appears to be a mandatory step before a complete modeling can be proposed.

\section{CONCLUSIONS}

The impact of a droplet on a granular material has been investigated. The crater morphology has been characterized and two regimes have been evidenced according to the Weber number. The existence of these regimes originates from the competition between the characteristic time needed for the spreading and the time needed for the imbibition by the sand. From the dynamical point of view, the spreading of the droplet occurs similarly to that of a droplet impacting a solid. The recession is due to capillary forces and ends because of the impregnation of the sand layer by the drop. The sand granulometry has been changed. When the grains become too large, recession is not observed: the energy provided by the impact is not sufficient to move the grains.

\section{ACKNOWLEDGMENTS}

SD thanks F.R.S.-FNRS for financial support. GD benefits from a BELSPO PRODEX program grant. This work is financially supported by ESA through MAP Contract No. AO-99-108 and FNRS-FRS Contract No. FRFC 2.4558.10.
[1] A. M. Worthington, The Splash of a Drop (General Literature Committee, London, 1895).

[2] R. D. Deegan, P. Brunet, and J. Eggers, Nonlinearity 21, C1 (2008).

[3] R. Rioboo, C. Bauthier, J. Conti, M. Voué, and J. De Coninck, Exp. Fluids 35, 648 (2003).

[4] R. Bergmann, D. van der Meer, M. Stijnman, M. Sandtke, A. Prosperetti, and D. Lohse, Phys. Rev. Lett. 96, 154505 (2006).

[5] P. Taylor, Curr. Opin. Colloid Interface Sci. 16, 326 (2011).

[6] M. Reyssat, A. Pepin, F. Marty, Y. Chen, and D. Quéré, Europhys. Lett. 74, 306 (2006).

[7] M. Reyssat, J. M. Yeomans, and D. Quéré, Europhys. Lett. 81, 26006 (2008).

[8] J. M. Aristoff and J. W. M. Bush, J. Fluid Mech. 619, 45 (2009).

[9] D. Lohse, R. Bergmann, R. Mikkelsen, C. Zeilstra, D. van der Meer, M. Versluis, K. van der Weele, M. van der Hoef, and H. Kuipers, Phys. Rev. Lett. 93, 198003 (2004).
[10] S. Deboeuf, P. Gondret, and M. Rabaud, Phys. Rev. E 79, 041306 (2009).

[11] S. B. Daziel and M. D. Steaton, in Sedimentation and Sediment Transport, edited by A. Gyr and W. Kinzelbach (Kluwer, Dordrecht, 2003).

[12] H. Katsuragi, Phys. Rev. Lett. 104, 218001 (2010).

[13] A. Rozhkov, B. Prunet-Foch, and M. Vignes-Adler, Proc. R. Soc. London, Ser. A 466, 2897 (2010).

[14] T. Tate, Phil. Mag. 27, 176 (1864).

[15] C. Clanet, C. Beguin, D. Richard, and D. Quéré, J. Fluid Mech. 517, 199 (2004).

[16] See Supplemental Material at http://link.aps.org/supplemental/ 10.1103/PhysRevE.84.046320 for a video showing the three kinds of impact.

[17] I. Roisman, R. Rioboo, and C. Tropea, Proc. R. Soc. London, Ser. A 458, 1411 (2002).

[18] D. Bartolo, C. Josserand, and D. Bonn, J. Fluid Mech. 545, 329 (2005). 